\title{
Interventions for Pregnant Women against Malaria: A Mini Review
}

\author{
Ahmed Dahiru Balami', Salmiah Muhammad Said and NorAfiah Muhammad Zulkefli \\ Department of Community Health, Universiti Putra Malaysia, Malaysia \\ *Corresponding author: Dr. Ahmed Dahiru Balami, PhD, Department of Community Health, Faculty of \\ Medicine and Health Sciences, Universiti Putra Malaysia, 43400 UPM Serdang, Selangor Darul Ehsan, \\ Malaysia
}

Malaria remains highly endemic in sub-Saharan Africa [1], and about 25 million pregnancies are estimated to occur yearly in malaria-endemic regions of the sub-continent [2]. Several interventions aimed at improving knowledge, attitude, and practice of malaria prevention, have been studied among different groups, only a few of which were among pregnant women. A systematic review of educational interventions in sub-Saharan Africa which included studies which focused on ITN, IPTp, home-based management and environmental management revealed evidence of its effectiveness in improving these practices among the intervention group [3]. Amoran [4] had demonstrated that directly training caregivers could lead to improvements in knowledge, attitudes and practice of ITN use. Among refugee mothers, health education, facilitated by Vector Control Programme personnel and Voluntary Collaborators, also led to improvements in knowledge of malaria transmission and positive treatment seeking behaviours for fevers with chills [5]. In Iran, a health education intervention based on the Protection Motivation Theory had led to improvements in malaria prevention self-efficacy scores, but not preventive behaviour scores among households in the intervention group [6]. Net impregnation also significantly improved among households, following a health education intervention on malaria with provision of net impregnation services in Piron, Mali [7]. Among Home-Based Care workers in Limpopo, South Africa, enrolled into a three to four-week training under the Malaria Awareness Programme (MAP), those who had completed the full course had thrice the odds of having knowledge of malaria transmission and knowledge of malaria prevention compared to those who were not in the training, or those who had dropped out after just a week [8].

For pregnant women, sleeping under an insecticidetreated net (ITN) and receiving intermittent preventive treatment in pregnancy (IPTp) would be the ultimate aim of any preventive intervention, as they are the main preventive practices recommended by the World Health Organization [9]. We reviewed published studies on interventions for pregnant women against malaria. The aim was to ascertain the progress made so far, identify current gaps, and provide suggestions for policy makers and future researchers on possible ways forward.

Summaries of few intervention studies conducted among pregnant women are presented in Table 1, Table 2 and Table 3. A recent study in a health centre in north-eastern Nigeria revealed that a four-hour malaria health educational intervention based on the information-motivation-behavioural skills was effective in leading to an overall improvement in knowledge, motivation and behavioural skills by $12.75 \%(p<0.001), 8.55 \%(p<$ $0.001)$, and $6.350 \%(p<0.001)$ respectively [10]. Training of Community-Directed Distributors (CDDs) to give basic counselling to antenatal women, was effective in improving both ITN use and IPT uptake among the intervention group [11]. In another study in Bangladesh, Community Health Workers visiting households with at least one pregnant woman or an under-five child, to give health education on malaria, was followed byan increase in insecticidal net use to above $80 \%$ [12]. This study however makes it difficult to draw conclusions as it had no control group. 
Table 1: Malaria health education intervention for household members.

\begin{tabular}{|c|c|}
\hline Study & Balami, et al. 2019 [10] \\
\hline Location & Maiduguri, Nigeria \\
\hline Participants & 372 antenatal care attendees (186 intervention +186 control) \\
\hline Intervention: & a. Malaria health education intervention \\
\hline Contents & b. Information on malaria transmission, complications, and prevention (ITN and IPTp) \\
\hline Methods & $\begin{array}{l}\text { a. Lectures using power point projections } \\
\text { b. Brain storming sessions } \\
\text { c. Practical demonstration sessions }\end{array}$ \\
\hline Duration & Four hours \\
\hline Guiding theory & Information-motivation-behavioural skills \\
\hline Control : & Educational session on breastfeeding \\
\hline Methods & $\begin{array}{l}\text { a. Lectures with power point projections } \\
\text { b. Brain storming sessions } \\
\text { c. Practical demonstration sessions }\end{array}$ \\
\hline Duration & Four hours \\
\hline Guiding theory & None \\
\hline Dependent variables: & a. Knowledge, motivation and behavioural skills for malaria prevention \\
\hline Follow-up duration & 2 months and 4 months post-intervention \\
\hline \multicolumn{2}{|l|}{ Summary of results: } \\
\hline & Knowledge of malaria and malaria prevention in pregnancy $=12.75 \%$ \\
\hline & Motivation for malaria prevention in pregnancy $=8.55 \%$ \\
\hline & Behavioural skills for malaria prevention in pregnancy $=6.350 \%$ \\
\hline
\end{tabular}

Table 2: Malaria health education intervention for pregnant women.

\begin{tabular}{|c|c|}
\hline Study & Okeibunor, et al. 2011 [11] \\
\hline Location & Akwalbom, Nigeria \\
\hline Participants & Pregnant women: 1,280 and another 1,380 \\
\hline Intervention: & $\begin{array}{l}\text { Complementary community-directed intervention (CDI) } \\
\text { programme }\end{array}$ \\
\hline Contents & $\begin{array}{l}\text { Community-directed distributors (CDDs) were trained } \\
\text { a. To deliver ITNs and SP (IPTp1 and IPTp2) to pregnant women, and } \\
\text { b. To provide basic health counselling services. }\end{array}$ \\
\hline \multicolumn{2}{|l|}{ Methods } \\
\hline Duration & Each batch was trained for 5 hours (all in a single day) \\
\hline Guiding theory & None \\
\hline Control : & $\begin{array}{l}\text { a. No CDI programme } \\
\text { b. Provision of public health clinics with drugs, functional equipment and other } \\
\text { supplies such as tracking sheets } \\
\text { c. Training for health personnel on antenatal care }\end{array}$ \\
\hline Contents & - \\
\hline Methods & - \\
\hline Duration & 7 months \\
\hline Guiding theory & - \\
\hline Dependent variables: & $\begin{array}{l}\text { a. ITN use } \\
\text { b. IPT uptake (at least two doses) }\end{array}$ \\
\hline \multirow[t]{4}{*}{ Summary of results: } & ITN use \\
\hline & $\begin{array}{l}\text { Percentage increase for intervention group: } \\
8.5 \text { percentage points }(95 \% \mathrm{Cl}: 0.045-0.122, \mathrm{p} \text {-value }<0.001)\end{array}$ \\
\hline & IPT adherence \\
\hline & 35.3 percentage points $(95 \% \mathrm{Cl}: 0.280-0.425, \mathrm{p}$-value $<0.001)$ \\
\hline
\end{tabular}


Table 3: Malaria health education intervention for household members.

\begin{tabular}{|c|c|c|}
\hline Study & \multicolumn{2}{|l|}{ Ahmed, et al. 2011 [12] } \\
\hline Location & \multicolumn{2}{|c|}{ Thirteen malaria-endemic districts in Bangladesh } \\
\hline Participants & \multicolumn{2}{|c|}{$\begin{array}{l}3,760 \text { households and another } 7,895 \text { households (household must have at least one under } 5 \\
\text { child and/or a pregnant women) }\end{array}$} \\
\hline Intervention: & \multicolumn{2}{|c|}{$\begin{array}{l}\text { a. Malaria health education intervention, and } \\
\text { b. Long-lasting insecticidal nets }\end{array}$} \\
\hline Contents & $\begin{array}{l}\text { Information on: } \\
\begin{array}{l}\text { a. Malaria and it } \\
\text { b. Norms of use } \\
\text { wash and dry } \\
\text { c. Diagnosis (by } \\
\text { algorithm }\end{array}\end{array}$ & $\begin{array}{l}\text { nets (e.g., when to hang the net, where and how to } \\
\text { many washes in a year etc.) } \\
\text { est) and prompt treatment of malaria following an }\end{array}$ \\
\hline Methods & \multicolumn{2}{|c|}{$\begin{array}{l}\text { a. Each community health worker }(\mathrm{CHW}) \text { visits } 10 \text { households in a day to give health } \\
\text { education on malaria } \\
\text { b. Distribution of long lasting insecticidal nets to poor households by the } \mathrm{CHWs}\end{array}$} \\
\hline \multicolumn{3}{|l|}{ Duration } \\
\hline Guiding theory & \multicolumn{2}{|l|}{ None } \\
\hline Control : & \multicolumn{2}{|l|}{ No control group } \\
\hline Contents & \multicolumn{2}{|l|}{-} \\
\hline Methods & \multicolumn{2}{|l|}{-} \\
\hline Duration & \multicolumn{2}{|l|}{-} \\
\hline Guiding theory & \multicolumn{2}{|l|}{-} \\
\hline Dependent variables: & \multicolumn{2}{|c|}{$\begin{array}{l}\text { a. Knowledge of malaria and insecticidal bed nets } \\
\text { b. Insecticidal net ownership } \\
\text { c. Insecticidal net use }\end{array}$} \\
\hline Follow-up duration & \multicolumn{2}{|l|}{2 years } \\
\hline \multicolumn{3}{|l|}{ Summary of results: } \\
\hline & \multicolumn{2}{|c|}{ Percentage of pregnant women who slept under an LLIN/ITN } \\
\hline & South-eastern districts & North-eastern-districts \\
\hline & $\%$ change $=6.8$ & $\%$ change $=6.6$ \\
\hline
\end{tabular}

The cost implications as well as small effect sizes of the previous interventions do not seem encouraging enough to make governments adopt them. More effective interventions are thus needed. The intervention module in one of the previous studies [10] could however complementon-going routine health education given to pregnant women during their ANC. The target pregnant women need to be actively involved in all stages of the development of such interventions. Qualitative studies in the form of focus group discussion and key informant interviews would play a great role in identifying strategies which need to be incorporated into such studies. Furthermore, additional interventions directed at significant others need to be implemented, considering the important role they play in determining pregnant women's use of ITN [13]. Owing to its wide coverage and effectiveness in influencing behaviour [14], the mass media also provides a promising platform for not only disseminating such intervention programmes, but also actively engaging remotely-located participants.

\section{References}

1. World Health Organization (2017) World malaria report 2016.
2. (2015) Centers for Disease Control and Prevention Web site, authors. Malaria facts.

3. Awusu-Addo E, Awusu-Addo S (2014) Effectiveness of health education in community-based malaria prevention and control interventions in sub-Saharan Africa: A systematic review. Journal of Biology, Agriculture and Healthcare 3: 22-34.

4. Amoran OE (2013) Impact of health education intervention on malaria prevention practices among nursing mothers in rural communities in Nigeria. Niger Med J 54: 115-122.

5. Cropley L (2004) The effect of health education interventions on child malaria treatment-seeking practices among mothers in rural refugee villages in Belize, Central America. Health Promot Int 19: 445-452.

6. Ghahremani L, Faryabi R, Kaveh MH (2014) Effect of health education based on the protection motivation theory on malaria preventive behaviors in rural households of Kerman, Iran. Int J Prev Med 5: 463-471.

7. Rhee M, Sissoko M, Perry S, McFarland W, Parsonnet J, et al. (2005) Use of insecticide-treated nets (ITNs) following a malaria education intervention in Piron, Mali: A control trial with systematic allocation of households. Malar J 4: 35.

8. Cox SN, Guidera KE, Simon MJ, Nonyane BA, Brieger W, et al. (2018) Interactive malaria education intervention and its effect on community participant knowledge: The malaria 
awareness program in Vhembe district, Limpopo, South Africa. Int Quart of Comm Heal Educ 38: 147-158.

9. WHO (2004) A strategic framework for malaria prevention and control during pregnancy in the African region. World Health Organization; National Population Commission.

10. Balami AD, Said SM, Zulkefli NM, Bachok N, Audu B (2019) Effects of a health educational intervention on malaria knowledge, motivation, and behavioural skills: A randomized controlled trial. Malaria Journal 18: 41.

11. Okeibunor JC, Orji BC, Brieger W, Ishola G, Otolorin E, et al. (2011) Preventing malaria in pregnancy through community-directed interventions: Evidence from Akwa Ibom State, Nigeria. Malar J 10: 227.

12. Ahmed SM, Hossain S, Kabir MM, Roy S (2011) Free distribution of insecticidal bed nets improves possession and preferential use by households and is equitable: Findings from two cross-sectional surveys in thirteen malaria endemic districts of Bangladesh. Malar J 10: 357.

13. Balami AD, Said SM, Zulkefli NM, Bachok N, Audu B (2018) Knowledge, motivation, self-efficacy, and their association with insecticidal net use among pregnant women in a secondary health centre in Maiduguri, Nigeria. Malar J 17: 359.

14. Ankomah A, Adebayo SB, Arogundade ED, Anyanti J, Nwokolo E, et al. (2012) Determinants of insecticide-treated net ownership and utilization among pregnant women in Nigeria. BMC Public Health 12: 105. 